\title{
VARIAÇÕES GLACIO-EUSTÁTICAS RELACIONADAS AOS CICLOS DE MILANKOVITCH CONTROLANDO A SEDIMENTAÇÃO CARBONÁTICA DO MIOCENO SUPERIOR, NA PORÇÃO SUL DA BACIA DE SANTOS.
}

\author{
MARIA DO CARMO GARCIA SEVERINO ${ }^{1}$, ARMANDO ANTÔNIO SCARPARO CUNHA², \\ VALESCA BRASIL LEMOS ${ }^{3}$
}

\begin{abstract}
GLACIO-EUSTATIC VARIATIONS RELATED TO MILANKOVITCH CYCLE BAND CONTROLLING THE UPPER MIOCENE CARBONATE SEDIMENTATION IN THE SOUTHERN PART OF THE SANTOS BASIN. The geological record of orbital-driven cycles in a succession of the upper Miocene carbonate rocks present in the Santos Basin, sotheastern Brazil, were studied in detail using geophysical logs, seismic sections, isotopic and biostratigraphic data. Spectral analyses performed on gamma-ray revealed 5 cycles interpreted to be related to eccentricity $(100$ and $410 \mathrm{ka})$, the time span of that carbonated deposits were estimated $2.05 \mathrm{Ma}$ following an accumulation rate that ranges from 18.8 to $28.1 \mathrm{~cm} / \mathrm{ka}$. The accumulation rate changes were connected with the relative position of the wells on the Platform. These glacio-eustatic cycles are associated with a geological setting of relatively constant subsidence and sea level increase (Highstand Sistem Tract).
\end{abstract}

Keywords: Cyclostratigraphy, Carbonate sedimentation, glacio-eustatic cycles, Santos Basin.

Resumo O registro de ciclos de Milankovitch em uma sucessão sedimentar carbonática do Mioceno superior da Bacia de Santos foi estudado em detalhe por meio da integração de perfis geofísicos, dados sísmicos e bioestratigráficos. Através da análise espectral dos perfis de raios gama foi possível caracterizar 5 ciclos que foram interpretados como de excentricidade ( $410 \mathrm{e} 100 \mathrm{ka})$, estimando um período de 2,05 Ma para a sedimentação destes depósitos carbonáticos. As taxas de acumulação calculadas variam de $18,8 \mathrm{a} 28,1 \mathrm{~cm} / \mathrm{ka}$ e estão diretamente relacionadas às diferentes posições amostradas pelos poços na plataforma. Esses ciclos de origem glacio-eustática são interpretados como formados em um contexto geológico de subida relativa do nível do mar (Trato de Sistema de Mar Alto) e de subsidência relativamente constante.

Palavras-chave: Cicloestratigrafia, Sedimentação carbonática, ciclos glacio-eustáticos, Bacia de Santos.

INTRODUÇÃO Na porção sul da Bacia de Santos, durante o Mioceno, estabeleceram-se condições propícias ao desenvolvimento de uma sedimentação mista (carbonatos/siliciclásticos).

A integração de dados de sísmica de reflexão multicanal regional, perfis de poços, análise litofaciológica e datações, com base em nanofósseis calcários, levaram à definição de quatro superseqüências deposicionais (Ss) e ao estabelecimento de um modelo de evolução paleoambiental (Severino 2000).

O trato de sistema de mar alto (TSMA) da Ss do Mioceno médio/superior é caracterizado por uma expressiva sedimentação carbonática que levou à formação de uma extensa plataforma carbonática na porção norte da área de estudo e uma plataforma isolada no compartimento central. As áreas com controle de poços mostram depósitos carbonáticos com espessuras que variam de 300 a 650 metros.

Embora o intervalo Mioceno seja marcado por uma superposição de eventos de variação relativa do nível do mar, durante os estágios de mar elevado do Neomioceno, tanto nos perfis sísmicos como nos perfis de raios gama, este intervalo é o que apresenta as maiores evidências de ocorrência de seqüências deposicionais de mais alta freqüência.

Tal fato permitiu a realização de uma análise de ciclicidade, com o objetivo de definir se realmente os ciclos transgressivosregressivos observados nos dados correspondiam a variações relativas do nível do mar de mais alta freqüência e quais os fatores que teriam controlado sua formação. Além disso, os dados bioestratigráficos indicavam o desenvolvimento do TSMA nas biozonas NN-8/NN-11 do Mioceno superior/Plioceno (Martini 1971) abrangendo um intervalo temporal máximo de 5,5 Ma.
Se a deposição carbonática houvesse envolvido todo este intervalo de tempo com taxas de acumulação constantes, esta teria valores da ordem de $12 \mathrm{~cm} / \mathrm{ka}$, considerados bastante baixos para rochas carbonáticas (Rao 1996, Schlager 1981). O estudo de ciclicidade, realizado neste intervalo, permitiu estimar com maior precisão a duração do TSMA e calcular as taxas médias de acumulação carbonática.

METODOLOGIA A teoria de Milankovitch, ou de indução orbital do clima, parte do pressuposto de que o sistema climático terrestre se comporta de modo linear em relação às oscilações periódicas nos valores de insolação, geradas pela variação cíclica dos parâmetros orbitais. Se forem preservadas dentro do registro sedimentar, tais modificações ambientais definirão unidades litológicas rítmicas, cujas variações de espessura poderão expressar relações similares àquelas existentes entre os períodos conhecidos dos parâmetros orbitais da Terra: excentricidade, $410 \mathrm{ka} \mathrm{e} 100 \mathrm{ka}$; obliqüidade, $41 \mathrm{ka}$; e precessão, 23 ka e 19 ka (Einsele et al. 1991; De Boer \& Smith 1994; Schwarzacher 1993).

O registro de uma sucessão sedimentar, climaticamente induzida, será uma composição destes parâmetros, tornando-se necessária a aplicação de técnicas apropriadas para individualizá-los. O reconhecimento e individualização dos ciclos existentes podem ser feitos através da análise espectral de uma série temporal, elaborada a partir de qualquer propriedade que identifique o caráter oscilatório da sucessão sedimentar. Identificados os ciclos e determinados os seus comprimentos de onda, poderão ser estimados para cada conjunto de dados, os prováveis parâ-

1 - PETROBRAS - E\&P-Exploração - Av Chile, 65 - sala 1302 - Centro - 20031-912 - Rio de Janeiro, RJ, Brasil - carmogs@petrobras.com.br

2 - PETROBRAS - Centro de Pesquisas e Desenvolvimento - Av Jequitibá, 950 - Cidade Universitária - Ilha do Fundão - 21949-900 - Rio de Janeiro, RJ, Brasil -scarparo@petrobras.com.br

3 - UFRGS - Universidade Federal do Rio Grande do Sul - Av Bento Gonçalves, 9500, prédio 43.127, sala 211 - Campus do Vale - $91509-900$ - Porto Alegre, RS Brasil - valesca.lemos@ufrgs.br 


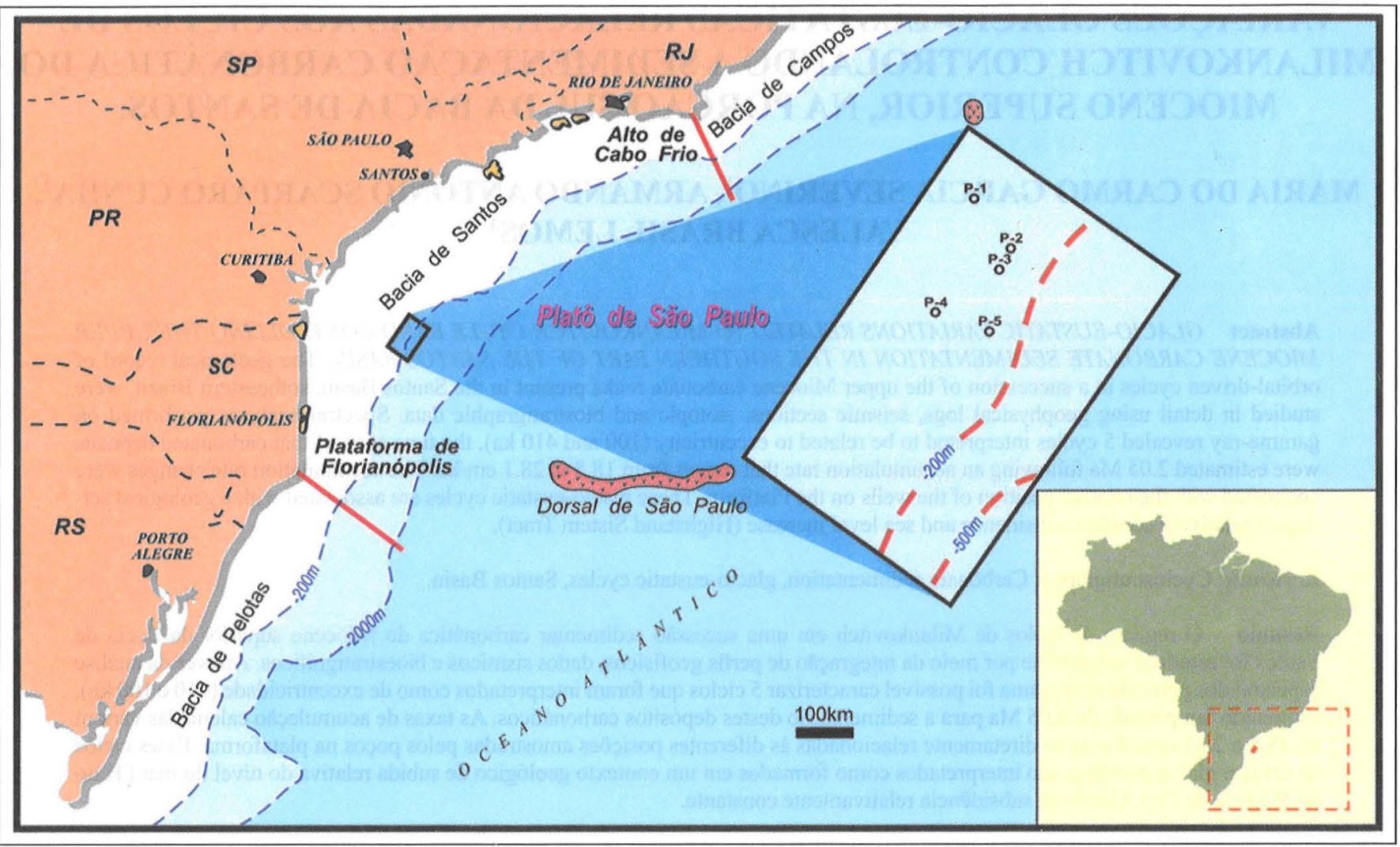

Figura 1 - Mapa de localização da Bacia de Santos com a identificação dos principais limites fisiográficos que a separam das bacias de Campos e Pelotas. A área de estudo está em destaque.

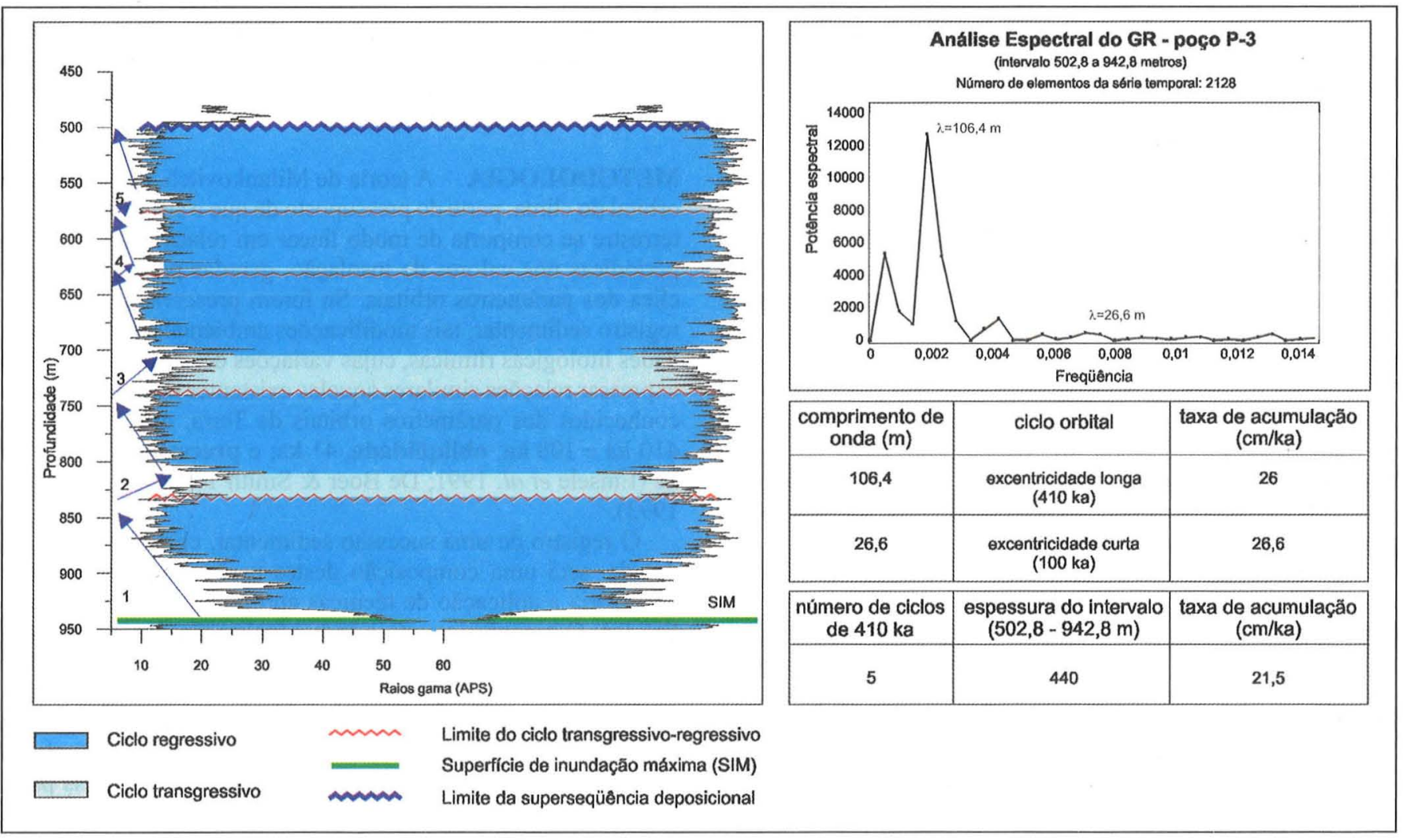

Figura 2 - Análise espectral, identificação dos ciclos transgressivos-regressivos no perfil de raios gama e cálculo das taxas de acumulação do poço P-3. O ciclo mais destacado no periodograma é o que apresenta comprimento de onda de 106,4 metros e corresponde à excentricidade longa $(410 \mathrm{ka}$ ). As taxas de acumulação foram recalculadas pelo número de ciclos identificados (cinco), obtendo-se taxas de acumulação média de $21,5 \mathrm{~cm} / \mathrm{ka}$. 
metros orbitais controladores dos alociclos. Para isso, pode-se utilizar uma técnica matemática denominada de similaridade de matrizes (Harbaugh \& Merriam 1968). Os aspectos conceituais, matemáticos e a aplicação deste método para quantificar a periodicidade em uma determinada sucessão cíclica de rochas foram amplamente discutidos em vários trabalhos (Hilgen \& Langereis 1989, Azambuja Filho 1990 e Azevedo et al. 1996).

Neste estudo foi utilizada a técnica de análise espectral nos perfis de raios gama dos poços que amostraram a seção carbonática do Mioceno superior, equivalente ao TSMA, na área plataformal (P-1, P-2, P-3, P-4 e P-5) (Fig. 1).

As leituras dos valores de raios gama, obtidas a cada $20 \mathrm{~cm}$, foram consideradas como sendo medidas a intervalos constantes de tempo. Ou seja, utilizou-se a profundidade como um parâmetro de tempo. Isto somente é possível ao assumir-se que a taxa de acumulação foi constante e que não existiram hiatos significativos no intervalo analisado.

Para a análise espectral foi utilizado o método da Transformada Rápida de Fourier e o programa utilizado para a análise espectral foi o programa STATISTICA (versão 4.3), que apresenta os resultados na forma de periodogramas.

Utilizando-se a técnica de similaridade de matrizes aplicada por Azambuja Filho (1990) em estudos cicloestratigráficos, comparou-se a razão dos valores dos comprimentos de ondas destacados no periodograma com os períodos orbitais de Milankovitch, utilizando-se uma similaridade de 90\%. Posteriormente, as taxas de acumulação foram recalculadas através da medição direta da espessura dos ciclos no perfil de raio gama.

RESULTADOS DA ANÁLISE ESPECTRAL A análise espectral realizada nos poços P-3, P-4 e P-5 comprovou a exis- tência de ciclos com um período bem definido, em torno de 100 metros, interpretado como correspondente à excentricidade longa (410 ka).

No poço P-3 o periodograma obtido da análise espectral mostra um pico principal, relacionado a um ciclo de 106,4 metros, e vários ciclos secundários (Fig. 2).

Das possibilidades de correlação apresentadas pelos cálculos matemáticos, verifica-se que apenas os ciclos de 106,4 metros e o de 26,6 metros, correspondendo aos ciclos de Milankovitch de $410 \mathrm{ka}$ (excentricidade longa) e $100 \mathrm{ka}$ (excentricidade curta), apresentaram taxas de acumulação compatíveis com as medidas obtidas em ambientes deposicionais análogos. Os valores médios considerados para o desenvolvimento de plataformas carbonáticas situam-se entre 20 a $30 \mathrm{~cm} / \mathrm{ka}$ (Schlager 1981), embora as taxas de sedimentação identificadas em plataformas carbonáticas atuais sejam extremamente variáveis (Rao 1996).

Para o ciclo de 106,4 metros foi calculada uma taxa de acumulação de $26 \mathrm{~cm} / \mathrm{ka}$ e para o ciclo de 26,6 metros, uma taxa de acumulação de $26,6 \mathrm{~cm} / \mathrm{ka}$. Qualquer outra interpretação que relacionasse o ciclo de 106,4 metros a um ciclo orbital de tempo inferior a $410 \mathrm{ka}$, como por exemplo o ciclo de excentricidade curta (100 ka), implicaria em taxas sedimentares muito elevadas. Além disso, considerando a existência de cinco ciclos, o intervalo temporal envolvido para o desenvolvimento desta sedimentação carbonática seria muito pequeno (em torno de $500 \mathrm{ka}$ ), não sendo compatível com os dados bioestratigráficos. Atribuindo uma duração total de 0,5 Ma para a deposição desta plataforma, o hiato temporal envolvido seria muito amplo, não sendo compatível com a interpretação dos demais dados que não mostram quebras acentuadas na sedimentação. A espessura do intervalo analisado (440 metros) dividido pela estimativa temporal $(2,05$

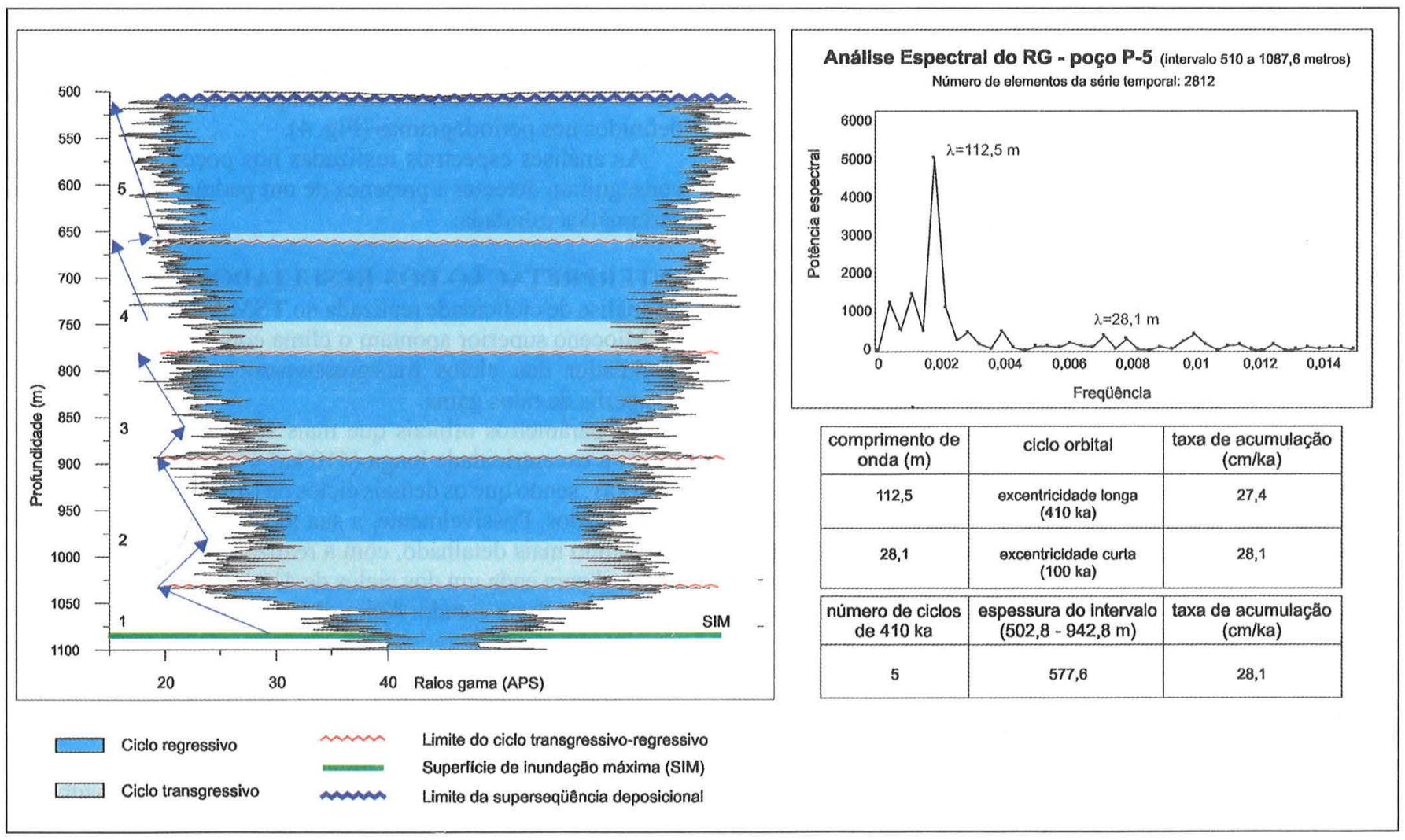

Figura 3 - Análise espectral, identificação dos ciclos transgressivos-regressivos no perfil de raios gama e cálculo das taxas de acumulação para o poço P-5. O ciclo mais destacado no periodograma é o que apresenta comprimento de onda de 112,5 metros, correspondendo à excentricidade longa $(410 \mathrm{ka}$ ). Identificados os ciclos no perfil de raios gama (cinco), as taxas de acumulação foram recalculadas obtendo-se valores médios de $28,1 \mathrm{~cm} / \mathrm{ka}$. 


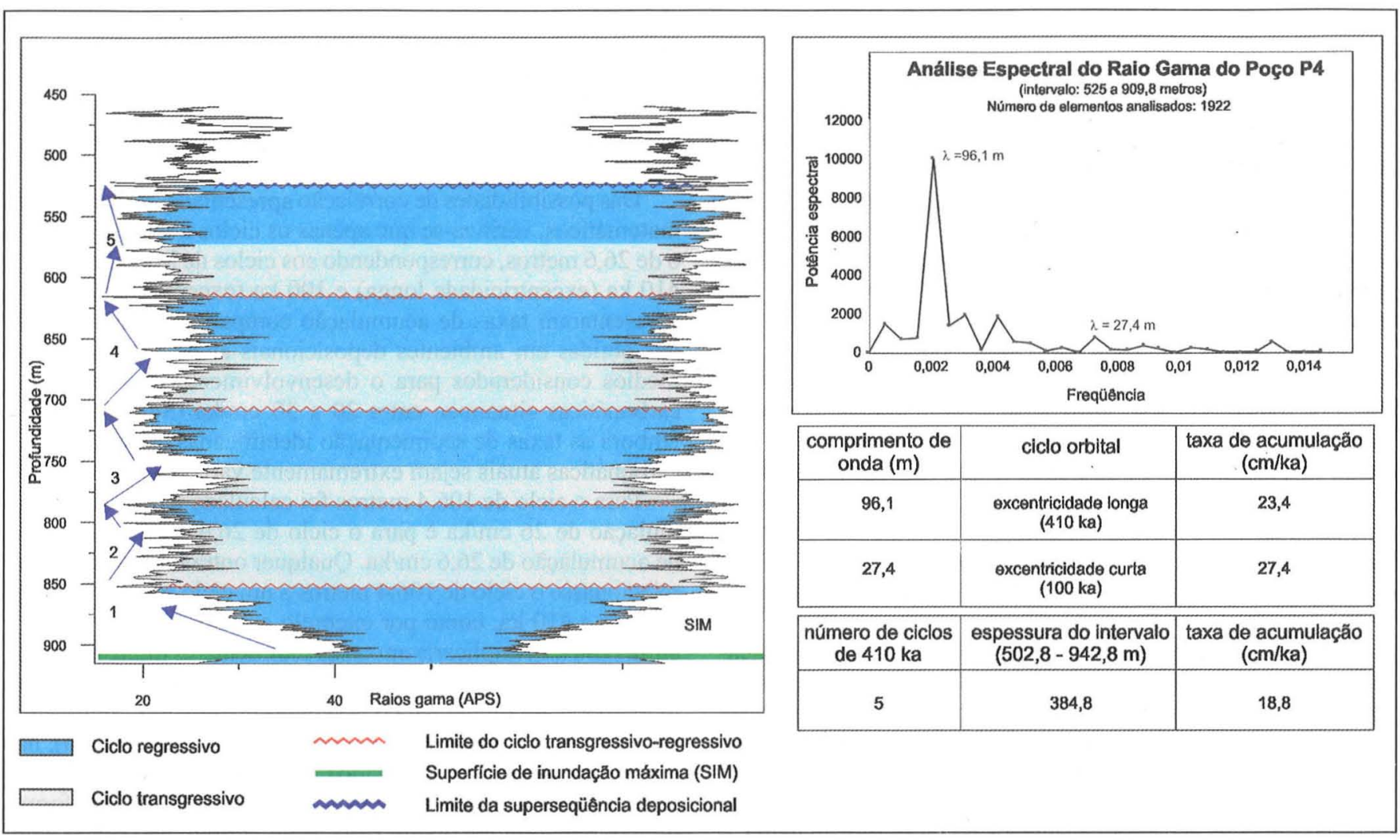

Figura 4 - Análise espectral, identificação dos ciclos transgressivos-regressivos no perfil de raios gama e cálculo das taxas de acumulação do poço P-4. O ciclo mais destacado no periodograma é o que apresenta comprimento de onda de 96,1 metros e corresponde à excentricidade longa $(410 \mathrm{ka}$ ). Identificados os ciclos no perfil de raios gama (cinco) as taxas de acumulação foram recalculadas obtendo-se valores médios de $18,8 \mathrm{~cm} / \mathrm{ka}$.

Ma) permite um cálculo mais aproximado da taxa de acumulação média, que seria para o poço P-3 de $21,5 \mathrm{~cm} / \mathrm{ka}$ (Fig. 2).

A análise espectral do perfil de raios gama do poço $\mathbf{P}-5$ (mesmo intervalo estratigráfico do poço P-3) mostrou uma freqüência de valor espectral elevado para um ciclo de comprimento de onda de 112,5 metros, e vários ciclos secundários de valores espectrais menores e de freqüências mais elevadas (Fig. 3). Os resultados foram semelhantes ao poço P-3. O ciclo de comprimento de onda de 112,5 metros foi correlacionado com o ciclo de Milankovitch de $410 \mathrm{ka}$, com taxas de acumulação de $27,4 \mathrm{~cm} / \mathrm{ka}$ e o de 28,1 metros, com o ciclo de $100 \mathrm{ka}$ e taxa de acumulação de $28,1 \mathrm{~cm} / \mathrm{ka}$. O recálculo dos valores, considerando os cinco ciclos observados no intervalo de análise, resultou em uma taxa de acumulação de $28,1 \mathrm{~cm} / \mathrm{ka}$ para a área do poço P-5.

O intervalo analisado no poço $\mathbf{P}-4$ apresentou resultados semelhantes aos anteriores, com um ciclo bem destacado de 96,1 metros e vários outros ciclos menores (Fig. 4). Na comparação de matrizes, as correlações coerentes relacionam o comprimento de onda de 96,1 metros com o ciclo de $410 \mathrm{ka}$, com taxas de acumulação de $23,4 \mathrm{~cm} / \mathrm{ka}$ e o comprimento de onda de 27,4 metros com o ciclo de $100 \mathrm{ka}$, com taxas de acumulação média de 27,4 cm/ka (Fig. 4). Subdividindo o intervalo de análise pelo número de ciclos identificados, obtém-se uma taxa de acumulação bem inferior, cerca de $18,8 \mathrm{~cm} / \mathrm{ka}$. Assumindo que a espessura dos ciclos interpretados, através da análise do perfil de raios gama é correta e que a duração dos ciclos é de $410 \mathrm{ka}$, o valor de $18,8 \mathrm{~cm} / \mathrm{ka}$ é mais coerente do que os valores obtidos pela análise espectral. Interpreta-se que os valores mais elevados, apontados pela análise espectral, são devidos a problemas metodológicos. A análise espectral em séries temporais representativas de seções com variações relativamente acentuadas na taxa de acumulação nem sempre resulta em ciclos bem definidos nos periodogramas (Fig. 4).

As análises espectrais realizadas nos poços $\mathbf{P}-\mathbf{1}$ e $\mathbf{P}-\mathbf{2}$ não conseguiram detectar a presença de um padrão cíclico na seção carbonática estudada.

INTERPRETAÇ̃̃O DOS RESULTADOS Os resultados da análise de ciclicidade realizada no TSMA da Superseqüência do Mioceno superior apontam o clima como o principal agente modulador dos ciclos transgressivos-regressivos observados nos perfis de raios gama.

Os parâmetros orbitais que mais se destacaram nos dados foram a excentricidade longa (410 ka) e a excentricidade curta (100 ka), sendo que os demais ciclos de Milankovitch não foram identificados. Possivelmente, a sua identificação dependeria de um estudo mais detalhado, com a realização de uma análise individual para cada um dos ciclos de $410 \mathrm{ka}$, não constituindo-se no objetivo deste estudo. Além disso, os ciclos de obliqüidade usualmente são registrados preferencialmente em rochas formadas em altas latitudes (De Boer 1991).

Durante o Neomioceno o clima provavelmente foi o principal agente modulador do influxo de sedimentos continentais, na área de estudo, causando mudanças na argilosidade dos carbonatos, refletida nos perfis raios gama, segundo um padrão de ciclo orbital de $410 \mathrm{ka}$. Possivelmente, num contexto geológico onde a geração do espaço para a acomodação dos sedimentos ocorreu segundo taxas relativamente constantes, o que fez com que os ciclos de $410 \mathrm{ka}$ ficassem bem marcados nos perfis.

Os poços que não apresentaram um padrão cíclico no registro sedimentar, P-1 e P-2, encontram-se situados na porção 
mais ao norte da área de estudo (Fig. 5). Esta região está situada próxima à ocorrência de domos de sal, alguns deles em processo de ascensão até o Recente. Esta movimentação possivelmente provocou, nas áreas mais próximas a halocinese, um incremento variável no espaço de acomodação, o que não permitiu a identificação de ciclos bem definidos nos perfis raios gama.

Várias discussões tem sido levantadas no sentido de explicar de que forma o clima influenciaria nas modificações de argilosidade, já que tal fato é observado em várias épocas do registro geológico, tanto em períodos glaciais como em não glaciais. Em geral, para os períodos não glaciais, os ciclos de climas úmi- dos propiciariam uma maior entrada de sedimentos siliciclásticos, o que diminuiria em muito a produção carbonática de água rasa, tanto pela entrada de água meteórica no sistema, quanto pelas alterações na turbidez da água. Ao contrário, em períodos de climas secos e mais quentes a tendência seria aumentar em muito a produtividade carbonática (Fitchen 1997, Handford \& Loucks 1993, Tucker \& Wright 1990). Todavia, as variações na produtividade carbonática e na quantidade de argila introduzida no sistema deposicional estão mais relacionadas com a formação de ciclos de raseamento (shallowing upward), do que de seqüências de alta freqüência, uma vez que estes mecanismos

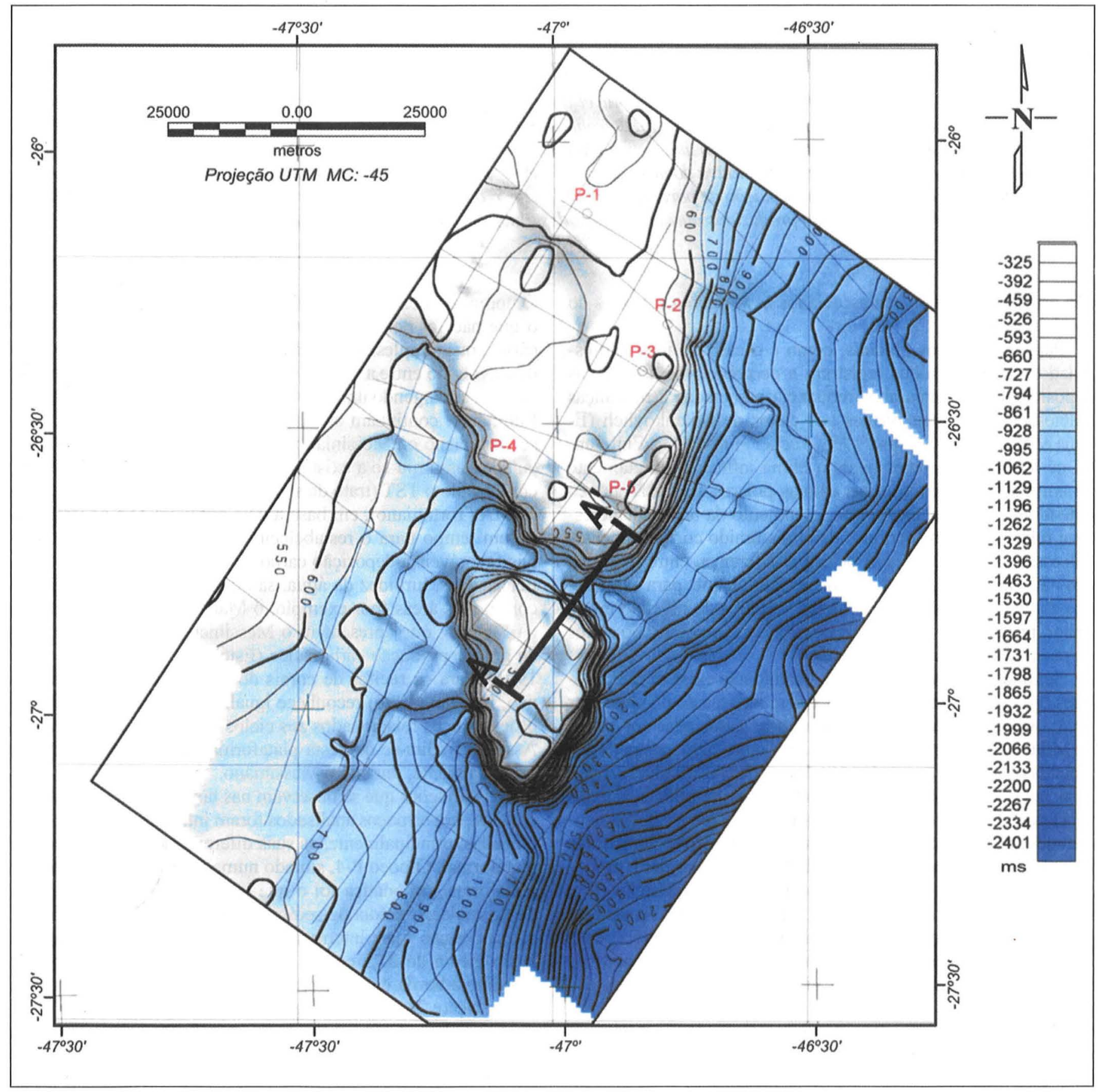

Figura 5 - Mapa estrutural sísmico do limite superior da Superseqüência do Mioceno médio/superior (Severino 2000). Durante o desenvolvimento do TSMA houve uma produção carbonática expressiva que culminou com a formação de uma ampla plataforma carbonática na porção norte e uma plataforma isolada na região central. As variações nas taxas de acumulação, calculadas para os poços $P-3(21,5 \mathrm{~cm} / \mathrm{ka}), P-4(18,8 \mathrm{~cm} / \mathrm{ka})$ e P-5 $(28,1 \mathrm{~cm} / \mathrm{ka})$, estão diretamente relacionadas às diferentes posições em que estes se encontram na plataforma. 

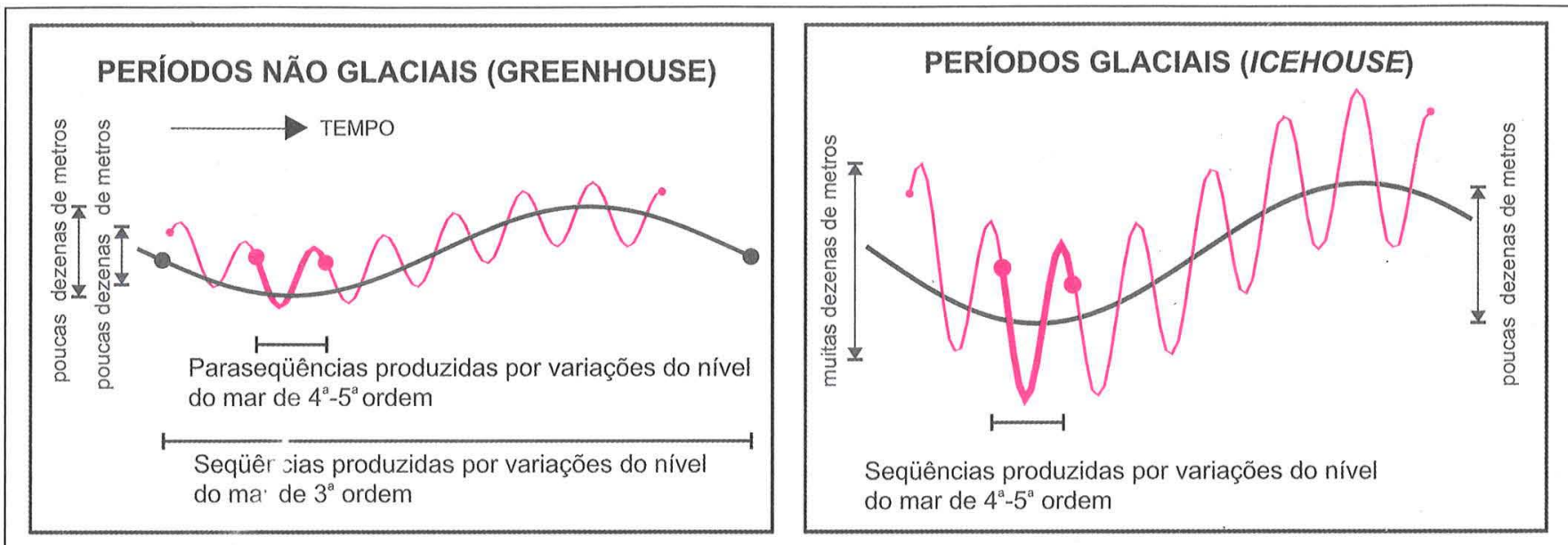

Figura 6 - Curvas esquemáticas de variação relativa do nível do mar de $3^{a}$ ordem e $4^{a} / 5^{a}$ ordem para os periodos não glaciais, greenhouse (a) e para os periodos glaciais, icehouse (b). Durante os períodos não glaciais, a curva de variação relativa do mar de $3^{a}$ ordem (1-10 Ma) possui uma amplitude maior do que a curva de variação relativa do nível do mar de $4^{a} / 5^{a}$ ordem (10 ka - $\left.1 \mathrm{Ma}\right)$. Para os períodos glaciais, a curva de variação relativa do nível do mar de $4^{a} / 5^{a}$ ordem possuem uma amplitude maior do que a de $3^{a}$ ordem. Assim, seqüências de $3^{a}$ ordem com formação de paraseqüências de $4^{a}$ e $5^{a}$ ordem são típicas para os períodos não glaciais; enquanto que seqüências de $4^{a}$ e $5^{a}$ ordem são produzidas durante os períodos glaciais (Modificado de Tucker et al. 1993).

não estão necessariamente associados às variações relativas do nível do mar.

Para os períodos glaciais, como é o caso do intervalo estudado, vários trabalhos registram a ocorrência de seqüências deposicionais de mais alta freqüência associadas a mudanças glacio-eustáticas relacionadas aos ciclos de Milankovitch (Evans \& Hine 1991, Esteban 1996, Tucker et al. 1993). Entretanto, os mecanismos pelos quais o clima influencia diretamente na ampliação ou redução das calotas polares ainda são pouco entendidos. De qualquer forma, o aumento da argilosidade estaria relacionado aos períodos de clima úmido correspondentes aos estágios interglaciais. A principal diferença, entre os períodos não glaciais e glaciais, reside no fato de que, para os períodos glaciais, a amplitude da variação relativa do nível do mar é maior, propiciando a formação de seqüências, em vez de ciclos de raseamento (shallowing upward).

Segundo Tucker et al. (1993), quando não existem capas polares (greenhouse) as amplitudes das mudanças do nível do mar de $3^{\text {a }}$ ordem são superiores às mudanças associadas aos ciclos de Milankovitch de $4^{\mathrm{a}}$ ou $5^{\mathrm{a}}$ ordem. Por outro lado, nos períodos glaciais (icehouse) as amplitudes das mudanças do nível do mar associadas aos ciclos climáticos orbitais são amplificadas pelos processos de formação das calotas polares. Neste caso, as amplitudes dos ciclos de $4^{\mathrm{a}}$ e $5^{\mathrm{a}}$ ordens freqüentemente são maiores que as variações relativas do nível do mar de $3^{a}$ ordem, produzindo sequiêencias de $4^{\mathrm{a}}$ e $5^{\mathrm{a}}$ ordens e não parasseqüiências (Fig. 6). Tal fato talvez explique a não identificação das seqüências de terceira ordem, no intervalo analisado; sendo possível apenas identificar os ciclos transgressivos-regressivos representativos de oscilações glacio-climáticas induzidas pelas variações dos parâmetros orbitais da Terra.

Corroborando com esta interpretação, os dados isotópicos de $\delta^{18} \mathrm{O}$ mostram uma clara tendência para valores mais positivos para o Meso e Neomioceno, indicando para este intervalo uma tendência geral de resfriamento (Severino 2000).

Em todos os perfis de raios gama analisados observa-se a presença de cinco ciclos bem marcados, permitindo sugerir que o intervalo de tempo de duração desta sedimentação carbonática foi de aproximadamente 2,05 milhões de anos (Figs. 2, 3 e 4). Este resultado possibilita inferir, com maior precisão, a duração temporal do desenvolvimento desta sedimentação carbonática, o que não foi possível através da datação de nanofósseis calcários, que simplesmente indicou que esta plataforma havia se desenvolvido entre a base da biozona NN-8 e a base da biozona $\mathrm{NN}-12$, abrangendo um intervalo de tempo em torno de 5,5 Ma. Entretanto, continuam as dúvidas sobre em qual dos andares, se Tortoniano ou Messiniano, ela foi desenvolvida (Fig. 7). No entanto, assumiu-se a existência de uma zona de condensação, englobando o TST (trato de sistema transgressivo) e o início do TSMA (Tortoniano), em base a dois critérios: 1) a necessidade de um tempo para o restabelecimento das condições ambientais favoráveis à deposição carbonática (recuo das fontes de siliciclásticos, turbidez da água, salinidade, etc.) e 2) correlação com outras áreas (por exemplo, o Mar Mediterrâneo e sudeste da Flórida), que apresentam o Messiniano como um intervalo de grande produção carbonática (Esteban 1996; Evans \& Hine 1991). Para os recifes de corais do Messiniano no Mediterrâneo, Esteban (1996) reconhece igualmente a ocorrência de padrões cíclicos relacionados aos ciclos orbitais de Milankovitch. Assim, acredita-se que esta plataforma tenha se desenvolvido, principalmente, durante o Messiniano.

As variações que se observam nas taxas de acumulação calculadas para os poços analisados foram interpretadas como relacionadas, principalmente, as suas diferentes posições na região plataformal. O poço $\mathrm{P}-4$, situado numa posição mais interna na plataforma carbonática foi o que apresentou as menores taxas de acumulação média de sedimentos: $18,8 \mathrm{~cm} / \mathrm{ka}$. O poço $\mathrm{P}-3$, numa posição mais intermediária apresentou taxas de acumulação média de $21,5 \mathrm{~cm} / \mathrm{ka}$. Já o poço $\mathrm{P}-5$, posicionado mais próximo à borda da plataforma apresentou as maiores taxas de acumulação: $28,1 \mathrm{~cm} / \mathrm{ka}$ (Fig. 5).

Em linhas gerais, as taxas de acumulação calculadas, para todo o intervalo de análise, seguem o padrão esperado: as menores taxas estão relacionadas às porções mais internas da plataforma e as maiores taxas localizadas próximo à borda da plataforma (Handford \& Loucks 1993).

Embora nenhum poço tenha sido analisado na porção central da área de estudo, interpreta-se nas linhas sísmicas também a ocorrência de cinco seqüências deposicionais, no mesmo intervalo estratigráfico da área em que se realizou a análise de 


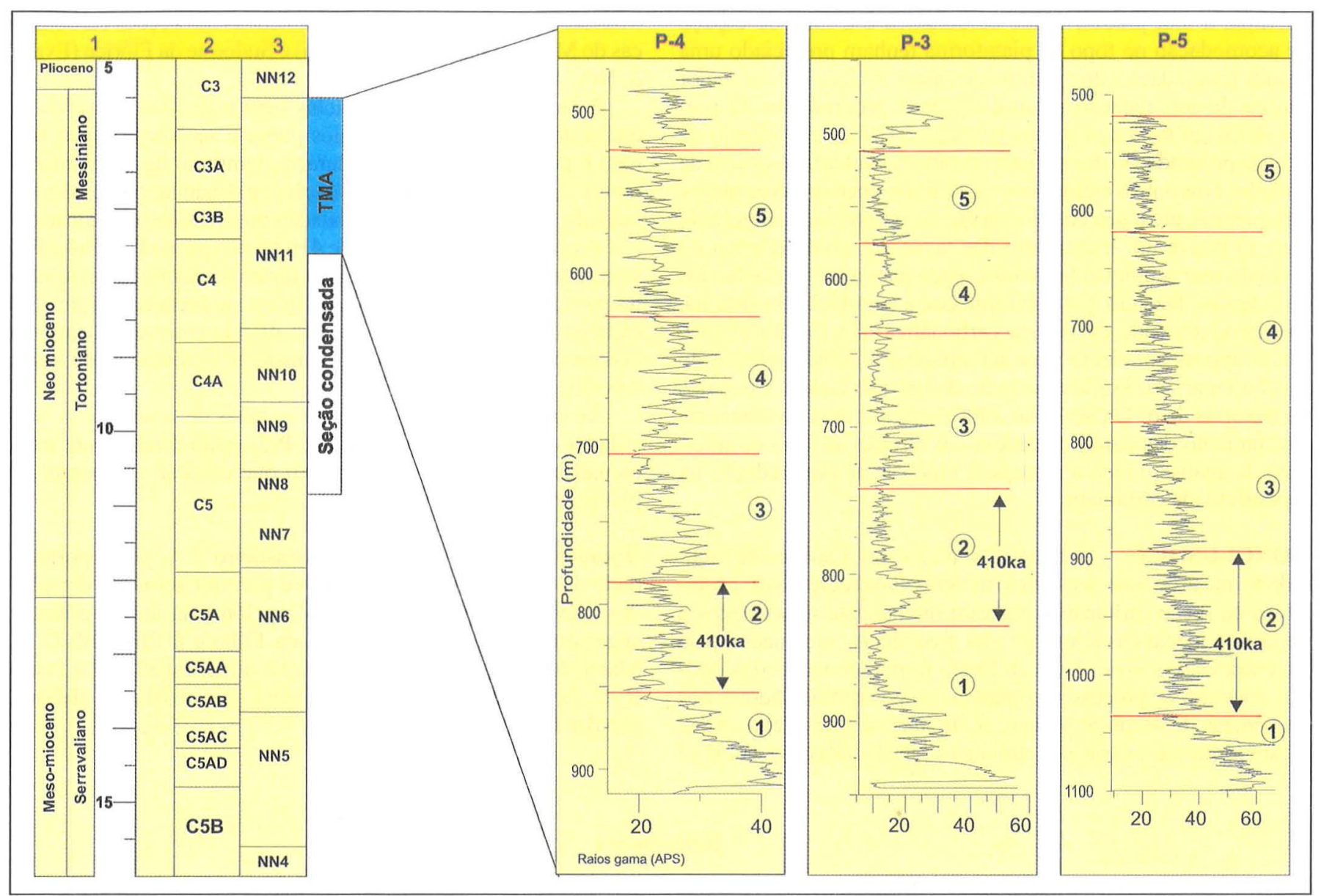

Figura 7 - Perfil esquemático posicionando o intervalo temporal atribuído ao TSMA da Superseqüência do Mioceno médio/superior pela datação dos nanofósseis calcários e pelos resultados da análise de ciclicidade, com cada ciclo transgressivo-regressivo envolvendo $410 \mathrm{ka}$. Nas colunas 1, 2 e 3 estão representadas, respectivamente: geocronologia com a escala de tempo e as unidades magnetoestratigráficas de Bergreen et al. (1985) e o zoneamento internacional de nanofósseis de Martini (1971).

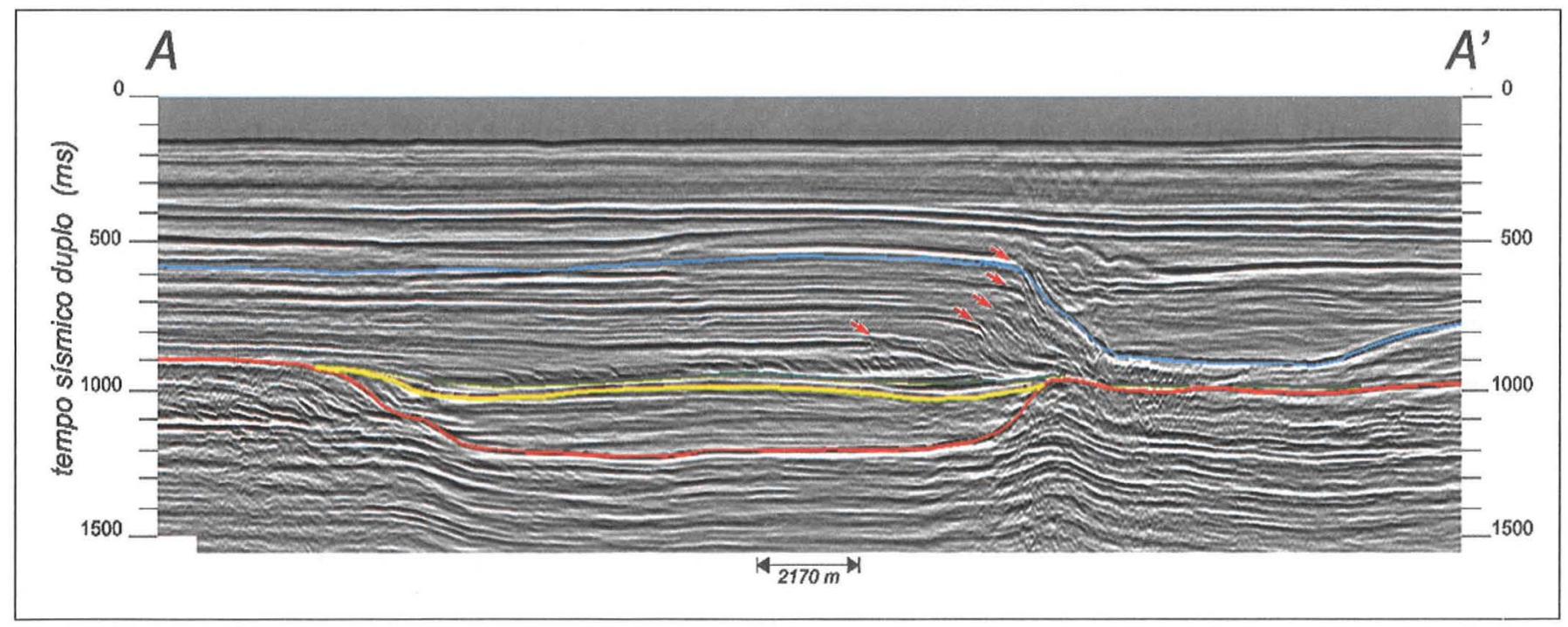

Figura 8 - Perfil sísmico com a interpretação da Ss do Mioceno médio/superior (Severino 2000). Durante o desenvolvimento do TSMA é possivvel identificar a ocorrência de cinco seqüências deposicionais de mais alta freqüência (setas vermelhas). A localização do perfil está na figura 5.

ciclicidade. Assim, pode-se considerar que cada uma destas seqüências foi depositada num intervalo de tempo de $410 \mathrm{ka}$, cor- respondendo a seqüências de $4^{\mathrm{a}}$ ordem (Fig. 8).

Nesta área, infere-se que logo após a superfície de inundação 
máxima, a alta produtividade carbonática e a falta de espaço de acomodação no topo da plataforma tenham propiciado uma rápida progradação dos sedimentos para os locais com maior espaço de acomodação. Assim, a grande progradação da quebra da plataforma deu-se nos primeiros 410 ka e o cálculo da taxa de progradação dos sedimentos atingiu valores na ordem de $30 \mathrm{~m} / \mathrm{ka}$. Nos outros ciclos observa-se um avanço cada vez menor das quebras, até o seu empilhamento no último ciclo, sugerindo que: 1) houve um incremento das taxas de subida relativa do nível do mar propiciando maior espaço para a acomodação dos sedimentos, fazendo que predominasse a agrađação do sistema; 2) a produtividade da fábrica carbonática não foi tão efetiva, sendo apenas suficiente para acompanhar a subida relativa do nível do mar; ou, 3) a inter-relação destes dois fatores. Os dados de isótopos de $\delta^{18} \mathrm{O}$ (Severino 2000) indicam uma tendência de resfriamento das águas oceânicas em direção ao topo do intervalo de análise, vindo a reforçar a hipótese de uma redução na produtividade carbonática.

CONCLUSÕES Durante o Neomioceno a análise de ciclicidade mostrou que os ciclos trangressivos/regressivos observados na seção carbonática estavam diretamente relacionados a variações glácio-eustáticas de alta freqüência, modulados pela excentricidade longa (410 ka). Desta forma, os resultados obtidos apontam os processos climáticos como os moduladores das seqüências de $4^{\mathrm{a}}$. ou $5^{\mathrm{a}}$. ordem, neste intervalo de tempo. Neste mesmo intervalo, variações climáticas relacionadas aos ciclos de
Milankovitch, foram também identificadas nas seções carbonáticas do Mediterrâneo (Esteban 1996) e Sudoeste da Flórida (Evans \& Hine 1991).

A identificação de cinco ciclos bem marcados em todos os dados analisados, perfis de raios gama e seções sísmicas, tornaram possível estimar uma duração temporal de aproximadamente 2,05 Ma para a sedimentação carbonática, o que não havia sido possível definir pela datação dos nanofósseis calcários.

A preservação dos ciclos de $410 \mathrm{ka}$ nos poços P-3, P-4 e P-5 sugere que as taxas de subsidência foram relativamente constantes para estas áreas. Interpretou-se que a ausência destes padrões cíclicos nos poços P-1 e P-2 estão diretamente relacionados à ocorrência de taxas variáveis de subsidência devido a processos halocinéticos.

As variações encontradas para as taxas de acumulação, calculadas para os poços P-3, P-4 e P-5, estão diretamente relacionadas às diferentes posições em que estes se encontram na plataforma.

Agradecimentos À Petróleo Brasileiro S.A. pela oportunidade de desenvolver este trabalho e permitir a sua divulgação. Ao Clóvis F. Santos e Paulo R. Zarpelon pela leitura crítica e sugestões. Ao Nilo C. de Azambuja Filho e à Elizabeth C. V. Machado por incentivar a publicação deste trabalho. Ao Paulo R. C. Taveira e ao César F. de Oliveira pelo auxílio na elaboração das figuras.

\section{Referências}

Azambuja Filho N.C.de 1990. The oligo-miocene turbidites and associated facies of the Campos Basin, Offshore Brazil. Phd. Thesis, Imperial College of London, $456 \mathrm{p}$.

Azevedo R.L.M.de, Azambuja Filho N.C.de, Spadini A.R. \& Marçal R.A. 1996. Ciclicidade orbital marcando o registro sedimentar: um exemplo do Cretáceo "Médio" da Bacia de Campos, Brasil. In: Simpósio sobre o Cretáceo do Brasil, 4, Boletim de Resumos Expandidos, p. 33-38.

Berggren W.A., Kent D.V. \& Van Couvering A. 1985. The Neogene: Part 2. Neogene geochronology and chronostratigraphy. In: N. Snelling (ed.) The Chronology of the Geological Record. Boulder, Geological Society of America, Memoir 10, pp: 211-247.

De Boer P.L. 1991. Pelagic black shale-carbonate rhythms: Orbital forcing and oceanographic response. In: G. Einsele, W. Ricken \& A. Seilacher (eds.) Cycles and Events in Stratigraphy. Berlin, SpringerVerlag, pp.: 63-78.

De Boer P.L. \& Smith D.G. 1994. Orbital Forcing and Cyclic Sequences. In: P.L De Boer. \& D.G. Smith (eds.) Orbital Forcing and Cyclic Sequences. Oxford: Blackwell, International Association of Sedimentologists, Special Publication 19:1-14.

Einsele G., Ricken W. \& Seilacher A. 1991. Cycles and Events in Stratigraphy - Basic Concepts and Terms. In: G. Einsele, W. Ricken \& A. Seilacher (eds.) Cycles and Events in Stratigraphy. Berlin, Springer-Verlag, pp.: 1-19.

Esteban M. 1996. An Overview of Miocene Reefs from Mediterranean Areas: general trends and facies models. In: E.K. Franseen; M. Esteban; W.C. Ward \& J.M. Rouchy (eds.) Models for Carbonate Stratigraphy from Miocene Reefs Complexes of Mediterranean Regions. Tulsa: SEPM, Concepts in Sedimentology and Paleontology, 5:3-53.
Evans M.W. \& Hine A.C. 1991. Late Neogene sequence stratigraphy of a carbonate-siliciclastic transition: Shouthwest Florida. Geological Society of America Bulletin, 103:679-699.

Fitchen W.M. 1997. Carbonate Sequence Stratigraphy and its Application to Hydrocarbon Exploration and Reservoir Development. In: I. Palaz \& K.J. Marfurt (eds.) Carbonate Seismology. Tulsa: Society of Exploration Geophysicists, Geophysical Developments Series, 6:121-178.

Handford C.R. \& Loucks R.G. 1993. Carbonate Depositional Sequences and Systems Tracts - Responses of Carbonate Platforms to Relative Sea-Level Changes. In: R.G. Loucks \& J.F. Sarg (eds.) Carbonate Sequence Stratigraphy: Recent Developments and Applications. Tulsa: AAPG, Memoir 57, pp.:3-41.

Harbaugh J.W. \& Merriam D.F. (Eds.) 1968. Computer Applications in Stratigraphic Analysis. John Wiley \& Sons Inc., London, 282 pp.

Hilgen F.J. \& Langereis C.G. 1989. Periodicities of $\mathrm{CaCO}_{3}$ cycles in the Pliocene of Sicily discrepancies with the quasi-periods of Earth's orbital cycles? Terra Nova, 1:409-415.

Martini, E. 1971. Standard Tertiaty and Quaternary calcareous nannoplankton zonation. In: A. Farinacci (ed.) Conference of Planktonic Microfossils, Roma, 1970. Proceedings... 2. pp.: 739785 .

Rao C. P. (Ed.) 1996. Modern Carbonates: Tropical, Temperate, Polar. Carbonates Publishing, Austrália, 206 pp.

Schlager W. 1981. The paradox of drowned reefs and carbonate platforms. Geological Society of America Bulletin, 92:197-211.

Schwarzacher W. (Ed.) 1993. Cyclostratigraphy and the Milankovitch Theory. Elservier, Amsterdam, Developments in Sedimentology 
$52,225 \mathrm{pp}$.

Severino M.C.G. 2000. Evolução da sedimentação carbonática/ siliciclástica miocênica na porção sul da Bacia de Santos, margem sudeste do Brasil. Dissertação de Mestrado em Geociências, Instituto de Geociências, Universidade Federal do Rio Grande do Sul, 183 p.

Tucker M.E. \& Wright V.P. (Eds.) 1990. Carbonate Sedimentology.
Blackwell Scientific Publications, Oxford, 482 pp.

Tucker M.E., Calvet F. \& Hunt D. 1993. Sequence stratigraphy of carbonate ramps: systems tracts, models and application to the Muschelkalk carbonate plataforms of eastern Spain. In: H.V. Posamentier, C.P. Summerhayes, B.U. Haq \& G.P. Allen (eds.) Sequence Stratigraphy and facies associations. International Association of Sedimentologists, Special Publication 18: 397-415. 